01

\title{
Амплитудно-частотные и фазово-частотные характеристики вынужденных колебаний нелинейного дробного осциллятора
}

\author{
(C) Р.И. Паровик
}

Институт космофизических исследований и распространения радиоволн ДВО РАН,

Паратунка, Россия

E-mail: romanparovik@gmail.com

Поступило в Редакцию 29 марта 2019г.

В окончательной редакции 11 апреля 2019г.

Принято к публикации 11 апреля 2019г.

Обосновывается связь амплитудно-частотных и фазово-частотных характеристик вынужденных колебаний нелинейного дробного осциллятора с порядками дробных производных, которые входят в его модельное уравнение. С помощью компьютерного моделирования показано, что порядки дробных производных связаны с добротностью колебательной системы. Уменьшение старшего порядка (,дробная“ инерция) приводит к уменьшению добротности, а уменьшение младшего порядка (,дробное“ трение) — к увеличению добротности. Проведено сопоставление численных расчетов амплитудно-частотных и фазово-частотных характеристик для линейного дробного осциллятора с расчетами по аналитическим формулам, полученным другими авторами. Анализ показал хорошее согласие, что подтверждает корректность представленных численных расчетов.

Ключевые слова: дробный осциллятор, добротность, амплитудно-частотная характеристика, фазовочастотная характеристика.

DOI: 10.21883/PJTF.2019.13.47953.17811

В рамках дробной динамики исследуются дробные или эредитарные осцилляторы - колебательные системы, обладающие эффектами степенной памяти [1-9]. Эти эффекты проявляются в том, что текущее состояние колебательной системы зависит от конечного числа предыдущих состояний. Такую нелокальность можно математически описать с помощью интегродифференциальных уравнений с разностными степенными ядрами - функциями памяти [10]. Однако более удобно, на наш взгляд, для дальнейшего исследования перейти от интегродифференциальных уравнений к уравнениям с производными дробных порядков, аппарат которых достаточно хорошо развит [11]. Здесь естественным образом может возникнуть вопрос о связи порядков дробных производных с характеристиками колебательного процесса, например с добротностью или декрементом затухания.

На этот вопрос был дан ответ в работе [9], где с помощью дробного исчисления и специальных функций Райта был проведен анализ вынужденных колебаний линейного дробного осциллятора без трения с внешним воздействием - аналога гармонического осциллятора. Этот анализ показал, что такая модель дробного осциллятора эквивалентна классической модели гармонического осциллятора с трением и внешним гармоническим воздействием, а порядок дробной старшей производной $\beta$, характеризующей ,дробную“ инерцию, связан с добротностью колебательной системы $Q$

$$
\beta \approx 2\left(1-\frac{1}{\pi Q}\right)
$$

В настоящей работе мы исследуем более общий нелинейный дробный осциллятор, описывающий вынужденные колебания, построим амплитудно-частотные и фазово-частотные характеристики (АЧХ и ФЧХ), установим связь между порядком старшей („дробная“ инерция) и младшей („дробное“ трение) дробных производных и добротностью системы $Q$. Для этого рассмотрим следующую задачу Коши:

$$
\begin{gathered}
\partial_{0 t}^{\beta} x(\eta)+\lambda \partial_{0 t}^{\gamma} x(\eta)+\omega^{\beta} \sin (x(t))=\delta \cos (\varphi t), \\
x(0)=x_{0}, \quad \dot{x}(0)=y_{0},
\end{gathered}
$$

где $x(t)$ - функция смещения, $t \in[0, T], T>0-$ время моделирования, $\lambda-$ коэффициент трения, $\omega-$ частота собственных колебаний, $\delta$ и $\varphi-$ амплитуда и частота внешнего периодического воздействия, $x_{0}$ и $y_{0}$ - константы, задающие начальные условия, а дифференциальные операторы

$$
\begin{gathered}
\partial_{0 t}^{\beta} x(\eta)=\frac{1}{\Gamma(2-\beta)} \int_{0}^{t} \frac{\ddot{x}(\eta) d \eta}{(t-\eta)^{\beta-1}}, \\
\partial_{0 t}^{\gamma} x(\eta)=\frac{1}{\Gamma(1-\gamma)} \int_{0}^{t} \frac{\dot{x}(\eta) d \eta}{(t-\eta)^{\gamma}},
\end{gathered}
$$

где

$$
1<\beta<2, \quad 0<\gamma<1,
$$

имеют смысл производных дробных порядков в смысле Герасимова-Капуто [12,13]. 
$a$

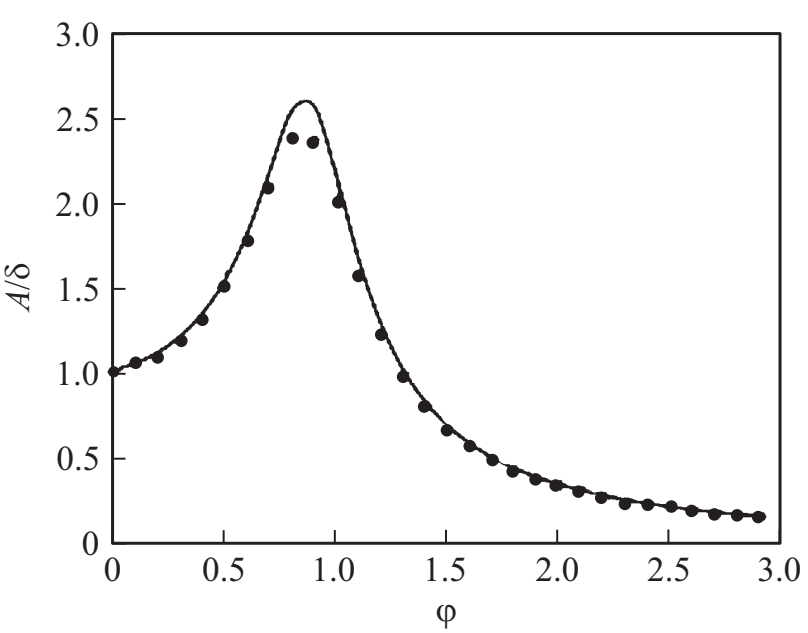

$c$

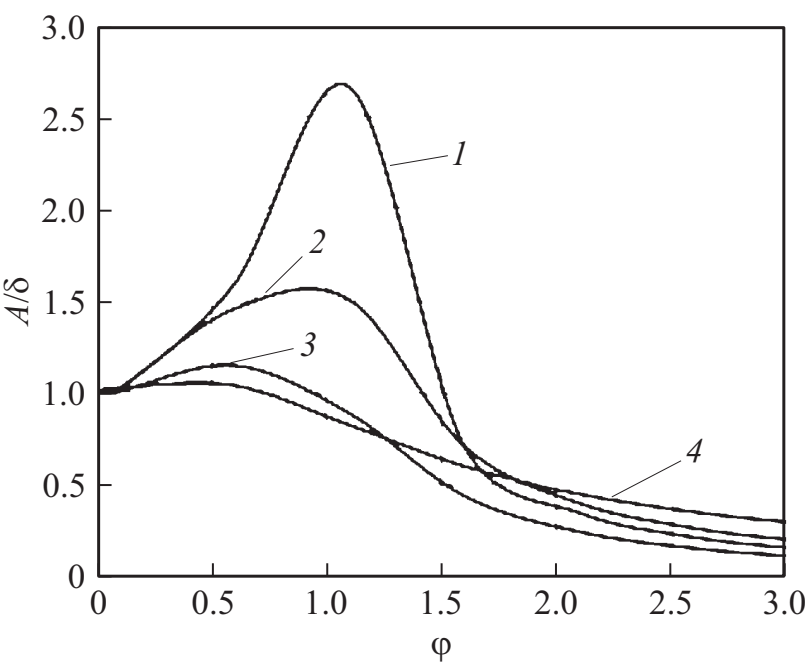

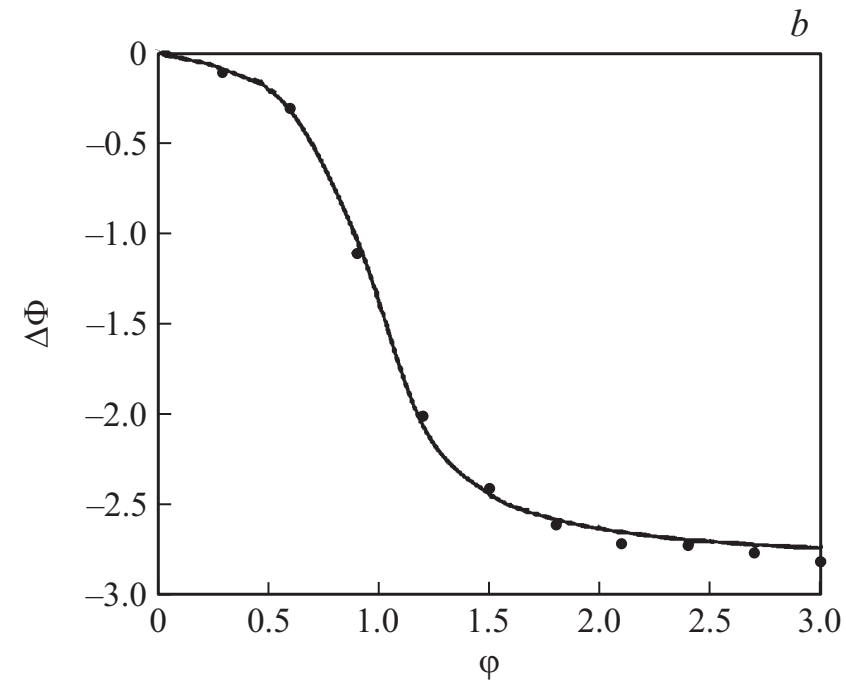

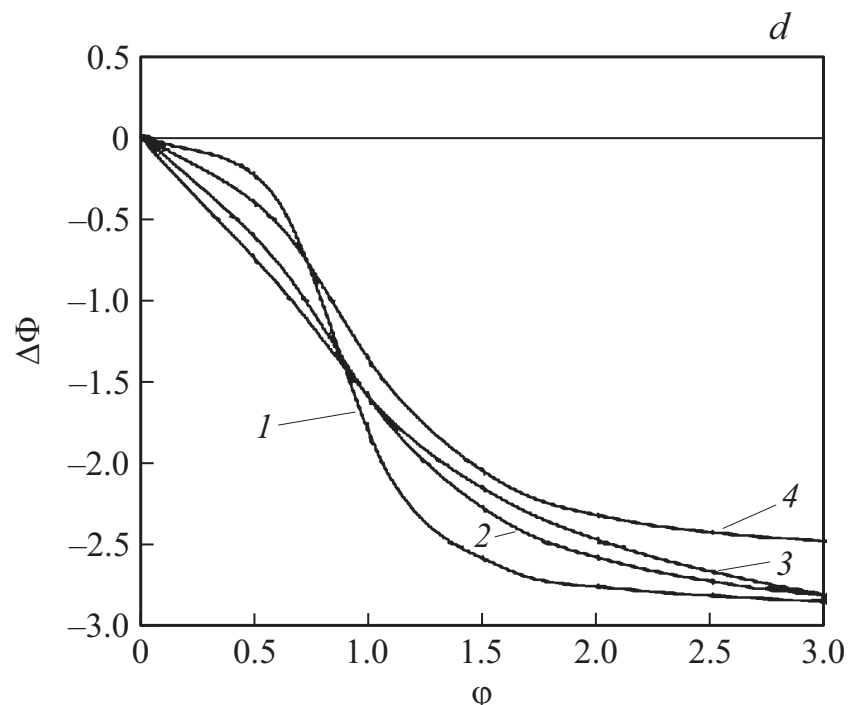

Рис. 1. $a-\mathrm{AЧX,} \mathrm{построенная} \mathrm{по} \mathrm{формуле} \mathrm{(14)} \mathrm{работы} \mathrm{[8]} \mathrm{(сплошная} \mathrm{линия),} \mathrm{точками} \mathrm{отмечена} \mathrm{АЧХ,} \mathrm{полученная} \mathrm{по}$

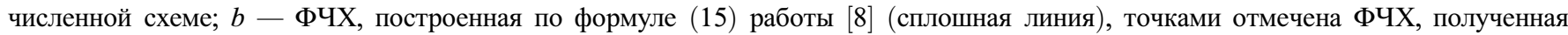
по численной схеме; $c-$ АЧХ для примера 1 : кривая $1-\beta=1.8, \lambda=0$, кривая $2-\beta=1.6, \lambda=0$, кривая $3-\beta=2, \lambda=1$, кривая $4-\beta=1.2, \lambda=0 ; d-$ ФЧХ для примера 1 : кривая $1-\beta=2, \lambda=0.5$, кривая $2-\beta=2, \lambda=1$, кривая $3-\beta=1.6$, $\lambda=0$, кривая $4-\beta=1.8, \lambda=0$.

Задача Коши (1) описывает дробный нелинейный осциллятор с „дробной“ инерцией и „дробным“ трением, а также внешним воздействием (вынужденные колебания). В случае малого угла смещения $\sin (x(t)) \approx x(t)$ получим дробный линейный осциллятор с внешним воздействием [9]. Необходимо отметить, что, как показано в работе [14], дробный нелинейный осциллятор (1) допускает хаотическую динамику, а в работе [15] были исследованы на устойчивость точки покоя.

Отметим, что, так как нелинейный дробный осциллятор (1) допускает хаотическую динамику, а нам необходимо исследовать регулярный установившийся режим, нужно выбирать соответствующим образом значения управляющих параметров согласно спектрам максимальных показателей Ляпунова [15]. АЧХ и ФЧХ вычисля- лись с помощью численного решения задачи Коши (1). Рассмотрим следующие примеры.

Пример 1: линейный дробный осциллятор [8,9]. Значения управляющих параметров возьмем следующими: $T=100, \omega=\gamma=1, \delta=10, x_{0}=0.2, y_{0}=0.3$.

На рис. 1, $a$ и $b$ приведены результаты расчета АЧХ и ФЧХ, полученные по формулам (14) и (15) из работы [8] (сплошные линии) и по численной схеме (точки), использованной нами. Видно, что численные расчеты хорошо аппроксимируют расчеты, полученные по аналитическим формулам из работы [8], что подтверждает корректность расчетов АЧХ и ФЧХ для примера 1.

Из рис. 1, $c$ видно, что при изменении значений дробного параметра $\beta \rightarrow 1$ резонансные кривые АЧХ становятся более пологими, а резонансная частота сме- 

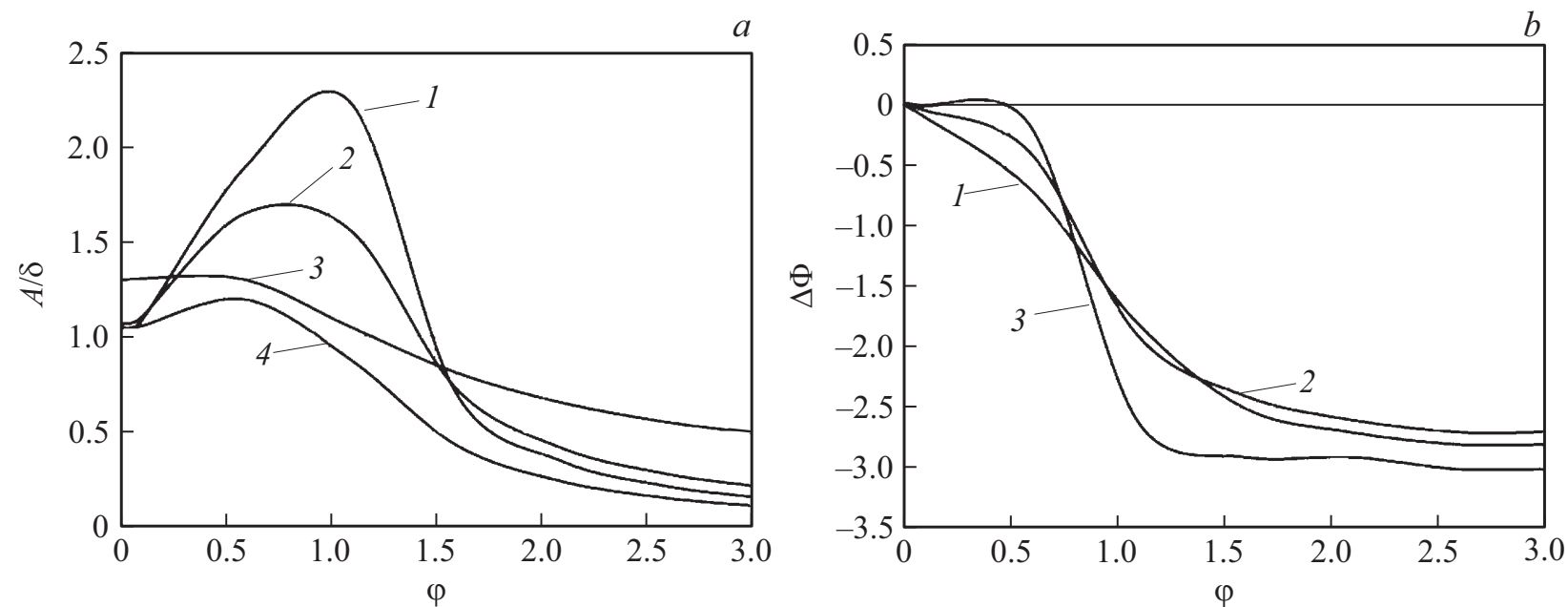

Рис. 2. $a-\mathrm{AЧX} \mathrm{для} \mathrm{примера} \mathrm{2:} \mathrm{кривая} 1-\beta=1.8, \lambda=0$, кривая $2-\beta=1.6, \lambda=0$, кривая $3-\beta=1.2, \lambda=0$, кривая $4-\beta=2, \lambda=1 ; b-$ ФЧХ для примера 2 : кривая $1-\beta=2, \lambda=1$, кривая $2-\beta=1.6, \lambda=0$, кривая $3-\beta=1.8$, $\lambda=0$. Частота $\varphi \in[0,3]$.
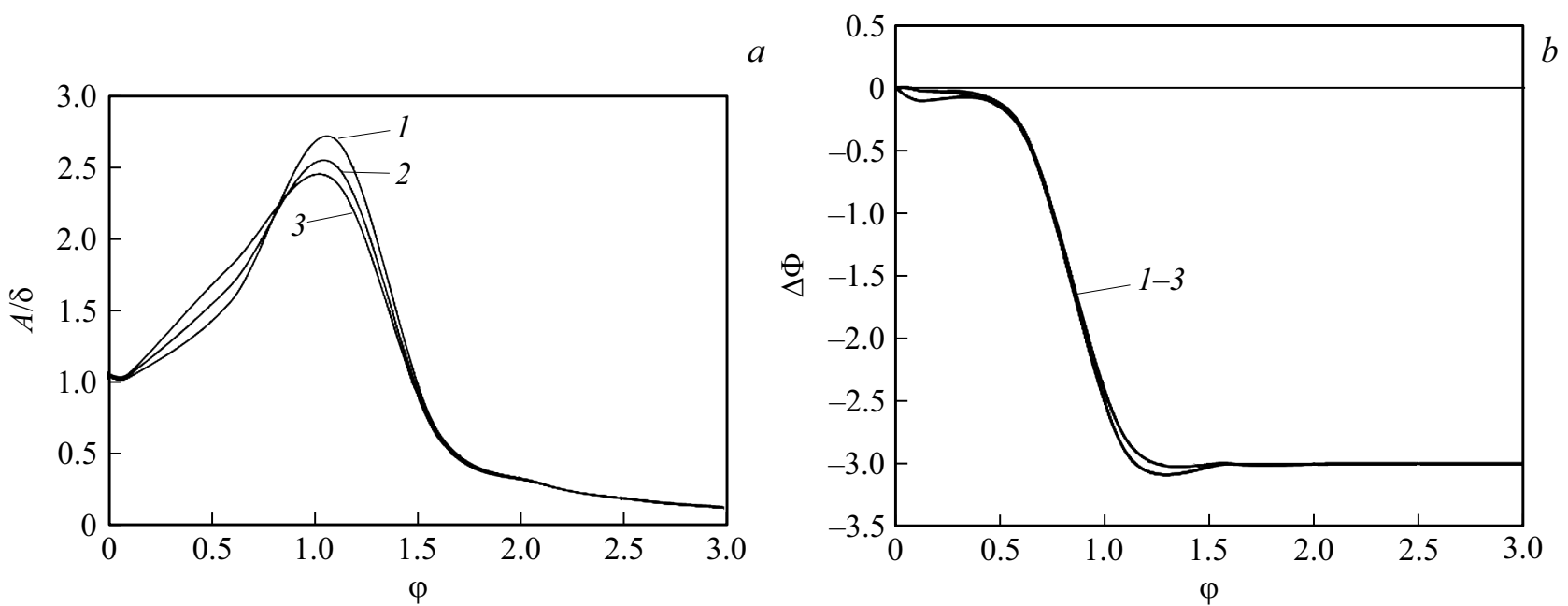

Рис. 3. АЧХ (a) и ФЧХ $(b)$ при $\beta=2$ и $\lambda=0.15 . \gamma=0.6(1), 0.8(2)$ и 1 (3). Частота $\varphi \in[0,3]$.

щается в область более низких частот. Такое поведение характерно для уменьшения добротности колебательной системы $Q$, так как отвечает за ширину резонансной кривой. Можно также обратить внимание на перегруппировку резонансных кривых, что характерно для эредитарных систем (или систем с памятью).

На рис. 1, $d$ приведены расчетные кривые ФЧХ. Отметим, что в работе [8] было указано на то, что при $\varphi \rightarrow \infty$ фазовый сдвиг $\Delta \Phi \rightarrow-\beta \pi / 2$. Как видно из рис. $1, d$,

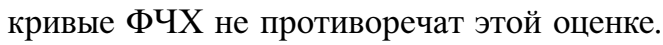

Пример 2: нелинейный дробный осциллятор. Значения управляющих параметров возьмем следующими: $T=200, \omega=\gamma=1, \delta=0.5, x_{0}=0.2, y_{0}=0.3$. Результаты моделирования приведены на рис. 2 .

Из рис. 2, а также видно, что при изменении значения $\beta \rightarrow 1$ резонансные кривые АЧХ становятся более пологими и резонансная частота смещается в область более низких частот, чему соответствует уменьшение добротности $Q$. Теперь рассмотрим, как влияет другой дробный параметр $(\gamma)$ при младшей производной, отвечающей за „дробное“ трение в уравнении (1). Построим АЧХ и ФЧХ при различных значениях $\gamma$ (рис. 3).

Видно (рис. $3, a$ ), что при уменьшении значений $\gamma \rightarrow 0$ резонансные кривые приобретают более пикообразную форму, о чем свидетельствует увеличение добротности $Q$ системы. Кривые ФЧХ на рис. $3, b$ почти совпадают; следовательно, параметр $\gamma$ практически не влияет на фазовый сдвиг $\Delta \Phi$.

Таким образом, можно сделать вывод, что изменение параметра $\beta \rightarrow 1$ приводит к уменьшению добротности $Q$, а изменение параметра $\gamma \rightarrow 0-$ к увеличению добротности $Q$. Поэтому дробные порядки производных, входящих в уравнение (1), не просто определяют свойства среды (эффекты памяти или эредитарности), а свя- 
заны с характеристикой самой колебательной системы, с ее добротностью. Из результатов исследования следует, что дробные параметры $\beta$ и $\gamma$ являются дополнительными степенями свободы, с помощью которых можно управлять добротностью колебательной системы (1).

\section{Финансирование работы}

Работа выполнена при поддержке гранта Президента РФ МК-1152.2018.1.

\section{Список литературы}

[1] Мейланов Р.П., Янполов М.С. // Письма в ЖТФ. 2002. T. 28. B. 1. C. $67-73$.

[2] Паровик Р.И. // Компьютерные исследования и моделирование. 2015. Т. 7. № 5. С. 1001-1021.

[3] Niu J., Shen Y., Yang S., Li S. // Int. J. Non-Linear Mech. 2017. V. 92. P. 66-75. DOI: 10.1016/j.ijnonlinmec.2017.03.014

[4] Li M. // Symmetry. 2018. V. 10. N 2. P. 40. DOI: $10.3390 / \mathrm{sym} 10020040$

[5] Li S., Niu J., Li X. // Chin. Phys. B. 2018. V. 27. N 12. P. 120502. DOI: 10.1088/1674-1056/27/12/120502

[6] Parovik R.I. // Вестн. ЮУрГУ. Сер. Мат. моделирование и программирование. 2018. Т. 11. В. 2. С. 108-122. DOI: $10.14529 / \mathrm{mmp} 180209$

[7] Wang Y., An J.Y. // J. Low Freq. Noise Vibr. Active Control. First Published: August 28, 2018. DOI: $10.1177 / 1461348418795813$

[8] Olivar-Romero F., Rosas-Ortiz O. // J. Phys.: Conf. Ser. 2017. V. 839. N 1. P. 12010 . DOI :10.1088/1742-6596/839/1/012010

[9] Псху А.В., Рехвиашвили С.Ш. // Письма в ЖТФ. 2019. T. 45. B. 1. C. $34-37$. DOI: 10.21883/PJTF.2019.01.47154.17540

[10] Volterra $V$. Theory of functionals and of integral and integrodifferential equations. Courier Corp., 2005. 288 p.

[11] Kilbas A.A., Srivastava H.M., Trujillo J.J. Theory and applications of fractional differential equations. Amsterdam: Elsevier, 2006. 523 p.

[12] Герасимов А.Н. // Прикладная математика и механика. 1948. T. 12. № 3. С. 529-539.

[13] Caputo M. Elasticità e dissipazione. Bologna: Zanichelli, 1969. $150 \mathrm{p}$.

[14] Паровик Р.И. Вестн. Самар. гос. техн. ун-та. Сер. Физ.-мат. науки. 2018. Т. 22. № 2. С. 364-379. DOI: $10.14498 /$ vsgtu1611

[15] Parovik R.I. // J. Phys.: Conf. Ser. 2018. V. 1141. P. 012079. DOI: $10.1088 / 1742-6596 / 1141 / 1 / 012079$ 